\title{
Analysis of photon-atom entanglement generated by Faraday rotation in a cavity
}

\author{
S. K. Y. Lee and C. K. Law \\ Department of Physics and Institute of Theoretical Physics, \\ The Chinese University of Hong Kong, Shatin, Hong Kong SAR, China
}

(Dated: July 11, 2018)

\begin{abstract}
Faraday rotation based on AC Stark shifts is a mechanism that can entangle the polarization states of photons and atoms. We study the entanglement dynamics inside an optical cavity, and characterize the photon-atom entanglement by using the Schmidt decomposition method. The timedependence of entanglement entropy and the effective Schmidt number are examined. We show that the entanglement can be enhanced by the cavity, and the entanglement entropy can be controlled by the initial fluctuations of atoms and photons.
\end{abstract}

PACS numbers: 03.67.Mn, 42.50.-p, 32.80.-t

The interaction between quantized optical fields and atomic ensembles provides physical models for exploring and realizing novel applications in quantum communication. A useful mechanism is the AC Stark shifts of offresonant light with two polarizations. As the frequency shift in each polarization is conditioned by the number of atoms at the corresponding magnetic sub-levels, any imbalance of atom numbers would lead to a rotation of Stokes parameters of light. The effect can be considered as a kind of Faraday rotation that correlates the polarization variables of photons and atoms, and it has been investigated theoretically and experimentally in the context of QND measurements and spin squeezing [1, 2, 3]. Interesting applications based on the interaction have also been discussed in recent literature. These include, for examples, quantum states teleportation and swapping [4], quantum memory [5], generation of macroscopic superposition states 6], and the implementation of Deutsch-Jozsa algorithm 7].

The key to most of these intriguing applications is the establishment of quantum entanglement between photons and atoms, governed by a model Hamiltonian [1]:

$$
H_{I}=2 g S_{z} J_{z}
$$

where $S_{z}$ is one of the Stokes operators of the field, $J_{z}$ is a collective spin operator of atoms, and $g$ is a coupling constant. Such an interspecies entanglement can be further converted into non-classical spin states by projective measurement of the light field. Previous studies of the interaction (1) have emphasized the quantum correlations of observables relevant to QND experiments 1, 2, 3]. From the view point of quantum information, a better understanding of entanglement can be gained by analyzing directly the photon-atom state vector in the Hilbert space. In particular, it would be useful to quantify the degree of entanglement and determine what parameters controlling the entanglement dynamics.

In this paper we approach the problem by studying the Schmidt decomposition of entangled states generated by (1). The distribution of Schmidt eigenvalues and the structure of Schmidt eigen-modes provide a complete

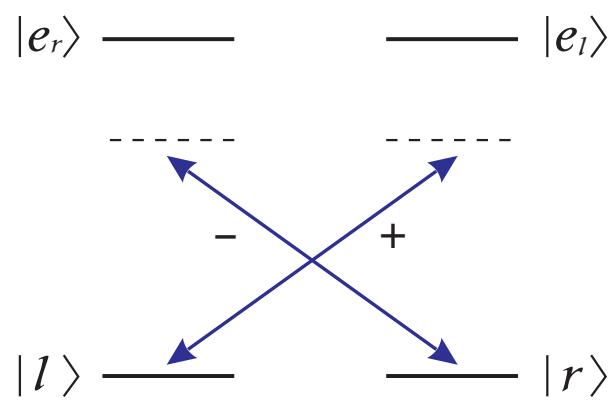

FIG. 1: (Color online) Atomic levels and interaction scheme of our model Hamiltonian. AC Stark shift is induced by nonresonant coupling between ground state $|l\rangle(|r\rangle)$ and polarization mode $+(-)$. For example, $|l\rangle$ and $|r\rangle$ refer to the two ground states with magnetic quantum numbers $\pm 1 / 2$.

characterization of pure-state entanglement [8]. In contrast to usual investigations in free space, we will examine the interaction (1) in an optical cavity. This is motivated by the fact that a strong atom-field coupling is often desirable to overcome decoherence. In free space, although the coupling strength can be increased at frequencies near resonances, the higher excitation rate would lead to substantial decoherence from spontaneous emission [3, 9]. The employment of a cavity provides a way to enhance the coupling strength while keeping the fields far away from resonance. In addition, the strong coupling may also allow us to explore cavity QED effects with fewer number of photons and atoms.

Our system consists of $N_{A}$ four-level atoms interacting with a quantized cavity field with two polarizations. The two degenerate atomic ground states are denoted by $|l\rangle$ and $|r\rangle$, and they are coupled to the excited states $\left|e_{l}\right\rangle$ and $\left|e_{r}\right\rangle$ by absorbing or emitting a photon of the corresponding polarization (Fig. 1). In our scheme, the field frequency is significantly detuned from the atomic transition frequency, so that the excited states are rarely populated. Hence the decoherence effects due to spontaneous decay from $\left|e_{l}\right\rangle$ and $\left|e_{r}\right\rangle$ can be safely neglected. The off-resonant interaction leads to AC Stark shifts, which 
are described by the effective Hamiltonian []]:

$$
H=g \sum_{k=1}^{N_{A}}\left(a_{+}^{\dagger} a_{+}|l\rangle_{k k}\left\langle l\left|+a_{-}^{\dagger} a_{-}\right| r\right\rangle_{k k}\langle r|\right)
$$

with $a_{+}$and $a_{-}$being the annihilation operators of the + and - polarizations of the cavity field. The coupling constant $g=-\left|\Omega_{0}\right|^{2} / \Delta$ is obtained by adiabatic elimination of the excited levels in the large detuning limit, $\Delta \gg\left|\Omega_{0}\right|$, where $\left|\Omega_{0}\right|$ is the vacuum Rabi frequency defined by the cavity mode. In our model, the atom-field coupling strength $g$ is assumed to be the same for each atoms. A ring cavity supporting traveling wave modes is more suitable to realize our model. This is because $g$ depends on the absolute square of mode amplitude, a traveling wave mode would give roughly the same value of $g$ inside the ring cavity.

By letting $\sigma_{z}^{(k)}=|l\rangle_{k k}\langle l|-| r\rangle_{k k}\langle r|, J_{z}=\frac{1}{2} \sum_{k} \sigma_{z}^{(k)}$ and $S_{z}=\frac{1}{2}\left(a_{+}^{\dagger} a_{+}-a_{-}^{\dagger} a_{-}\right)$, the Hamiltonian (2) can be expressed as: $H=\frac{g}{2} N_{A}\left(a_{+}^{\dagger} a_{+}+a_{-}^{\dagger} a_{-}\right)+2 g S_{z} J_{z}$. Since the first term corresponds to a constant shift of field frequency for a fixed particle number, it can be eliminated in a rotating frame. Faraday rotation is described by the second term, which is the same as (1). Noticing that $J_{z}$ and $S_{z}$ are formally equivalent to angular momentum operators, the corresponding eigenvectors are defined by: $J_{z}|j, m\rangle=m|j, m\rangle, J^{2}|j, m\rangle=j(j+1)|j, m\rangle$, $S_{z}\left|\frac{s}{2}, \frac{n}{2}\right\rangle=\frac{n}{2}\left|\frac{s}{2}, \frac{n}{2}\right\rangle, S^{2}\left|\frac{s}{2}, \frac{n}{2}\right\rangle=\frac{s}{2}\left(\frac{s}{2}+1\right)\left|\frac{s}{2}, \frac{n}{2}\right\rangle$. Here $2 j=N_{A}$ is the number of atoms, which is fixed in our problem. The $n$ and $s$ are difference and sum of photon numbers in the two polarization modes respectively.

Initially, the system is in a product (disentangled) state:

$$
|\Psi(0)\rangle=\left(\sum_{m=-j}^{j} A_{m}|j, m\rangle\right) \otimes\left(\sum_{s=0}^{\infty} \sum_{n=-s}^{s}{ }^{\prime} P_{s, n}\left|\frac{s}{2}, \frac{n}{2}\right\rangle\right),
$$

where $A_{m}$ and $P_{s, n}$ are amplitudes describing the initial state of atoms and photons respectively, and the primed summation sign refers to the step size equal to 2, i.e., $n=-s,-s+2,-s+4, \ldots,+s$. Based on the Hamiltonian $(1)$, the state vector at time $t$ is given by,

$$
|\Psi(t)\rangle=\sum_{s=0}^{\infty} \sum_{n=-s}^{s} \sum_{m=-j}^{j} A_{m} P_{s, n} e^{-i g m n t}|j, m\rangle\left|\frac{s}{2}, \frac{n}{2}\right\rangle,
$$

where the phase factor $e^{-i g m n t}$ is responsible for the entanglement. Since the quantum number $s$ plays no role in the phase factor, it is convenient to define a field state $|n\rangle_{f}$ by summing $s$ at a given $n$,

$$
|n\rangle_{f} \equiv F_{n}^{-1} \sum_{s=|n|}^{\infty}{ }^{\prime} P_{s, n}\left|\frac{s}{2}, \frac{n}{2}\right\rangle \quad n=0,1,2, \ldots
$$

Here $F_{n}^{2}=\sum_{s=|n|}^{\infty}{ }^{\prime}\left|P_{s, n}\right|^{2}$ is a normalization factor. With some careful counting of states, we note that the $s$ summation in Eq. (5) refers to the step size equal to 2 (i.e., $s=|n|,|n|+2, \ldots)$. We also note that the orthogonality relation ${ }_{f}\left\langle n^{\prime} \mid n\right\rangle_{f}=\delta_{n^{\prime}, n}$ is satisfied.

The time-dependent state vector (4) can now be expressed in a compact form:

$$
|\Psi(t)\rangle=\sum_{n=-\infty}^{\infty} \sum_{m=-j}^{j} A_{m} F_{n} e^{-i g m n t}|m\rangle_{a}|n\rangle_{f},
$$

where $|m\rangle_{a}$ is a short notation for the atomic state $\left|j=N_{A} / 2, m\right\rangle$. The evolution of the system is periodic with the period $t=2 \pi / g$. However, we shall see below that a significant degree of quantum entanglement can be established in shorter time scale, which depends on the fluctuations of atom (photon) numbers in the each atomic (polarization) states.

To reveal the pairing structure of photon-atom entanglement in the state (6), it is customary to perform the Schmidt decomposition so that,

$$
|\Psi(t)\rangle=\sum_{k=0}^{\infty} \sqrt{\lambda}_{k}\left|u_{k}\right\rangle_{a}\left|v_{k}\right\rangle_{f}
$$

Here $\lambda_{k}$ are Schmidt eigenvalues, and $\left|u_{k}\right\rangle_{a}$ and $\left|v_{k}\right\rangle_{f}$ are Schmidt eigenmodes of atoms and photons respectively. Since the Schmidt modes are orthogonal, i.e., ${ }_{a}\left\langle u_{k^{\prime}} \mid u_{k}\right\rangle_{a}={ }_{f}\left\langle v_{k^{\prime}} \mid v_{k}\right\rangle_{f}=\delta_{k k^{\prime}}$, if the field is found in the state $\left|v_{k}\right\rangle$, then with certainty the atomic states must be in $\left|u_{k}\right\rangle$. In addition, the distribution of Schmidt eigenvalues provides a measure of the degree of entanglement. This can be quantified by the entanglement entropy $\mathcal{S}=-\sum_{k} \lambda_{k} \ln \lambda_{k}$, but a more convenient measure is the effective Schmidt number $\mathcal{K}$ defined by [10]

$$
\mathcal{K}=\left(\sum_{k} \lambda_{k}^{2}\right)^{-1} .
$$

$\mathcal{K}$ can be interpreted as the 'average' number Schmidt modes in the expansion. The higher the value of $\mathcal{K}$, the higher the entanglement, which shares similar features as the entropy.

The Schmidt decomposition can be carried out by diagonalizing the reduced density matrix of individual subsystems [8]. For general state vectors, however, one needs to perform the diagonalization numerically. In order to gain analytic insight about the properties of entanglement, we consider a class of states in which $A_{m}$ and $F_{n}$ are defined by gaussian functions, and in addition, these amplitudes change smoothly with $m$ and $n$ :

$$
\begin{aligned}
& A_{m}=\chi_{A} e^{-\left(m-m_{0}\right)^{2} / \sigma_{A}^{2}} \\
& F_{n}=\chi_{F} e^{-\left(n-n_{0}\right)^{2} / \sigma_{F}^{2}} .
\end{aligned}
$$


Here $\chi_{A}$ and $\chi_{F}$ are normalization constants, and $m_{0}$ $\left(n_{0}\right)$ and $\sigma_{A}\left(\sigma_{F}\right)$ are parameters corresponding to the peak and width of atomic (photonic) amplitudes. In order to have $A_{m}$ described by a whole Gaussian in the range $-N_{A} / 2 \leq m \leq N_{A} / 2$, we require $N_{A} / 2>$ $2 \sigma_{A}+\left|m_{0}\right|$ so that $A_{ \pm N_{A} / 2}$ is negligible. We point out that the gaussian amplitudes (9) and (10) can be employed to capture or approximate a variety of states. These include atomic spin coherent states 11] and coherent states of photons 12 .

Now we make use of an identity derived from Mehler's formula,

$$
\sqrt{\frac{2}{\pi \sigma_{A} \sigma_{F}}} e^{-x^{2} / \sigma_{A}^{2}-y^{2} / \sigma_{F}^{2}} e^{-i g x y t}=\sqrt{1-\mu^{2}(t)} \sum_{k=0}^{\infty} \mu^{k}(t) U_{k}(x, t) V_{k}(y, t)
$$

where $U_{k}(x, t)$ and $V_{k}(y, t)$ are oscillator mode functions that can be expressed in terms of Hermite polynomial $H_{k}$ :

$$
\begin{aligned}
& U_{k}(x, t)=\sqrt{\frac{\xi}{\sigma_{A} \sqrt{\pi} 2^{k} k !}}(-i)^{k / 2} H_{k}\left(\xi x / \sigma_{A}\right) e^{\left.-\xi^{2} x^{2} / 2 \sigma^{2} 12\right)} \\
& V_{k}(y, t)=\sqrt{\frac{\xi}{\sigma_{F} \sqrt{\pi} 2^{k} k !}}(-i)^{k / 2} H_{k}\left(\xi y / \sigma_{F}\right) e^{-\xi^{2} y^{2} / 2 \sigma_{F}^{2}(13)}
\end{aligned}
$$

In writing Eq. (11), we have defined:

$$
\begin{aligned}
& \mu=\frac{\sqrt{1+\left(\sigma_{A} \sigma_{F} \tau\right)^{2}}-1}{\tau \sigma_{A} \sigma_{F}}, \\
& \xi=\sqrt{2}\left[1+\left(\sigma_{A} \sigma_{F} \tau\right)^{2}\right]^{1 / 4},
\end{aligned}
$$

with the dimensionless time $\tau \equiv g t / 2$.

With the help of Eq. (11), we treat $m \rightarrow x$ and $n \rightarrow$ $y$ and obtain an approximate Schmidt decomposition of the state Eq. (6). Specifically, we have the Schmidt eigenvalues

$$
\lambda_{k} \approx\left(1-\mu^{2}\right) \mu^{2 k}
$$

which decreases exponentially with mode index $k$, and the Schmidt eigenvectors,

$$
\begin{aligned}
\left|u_{k}\right\rangle_{a} & \approx \sum_{m=-j}^{j} U_{k}\left(m-m_{0}, t\right)|\tilde{m}\rangle_{a}, \\
\left|v_{k}\right\rangle_{f} & \approx \sum_{n=-\infty}^{\infty} V_{k}\left(n-n_{0}, t\right)|\tilde{n}\rangle_{f},
\end{aligned}
$$

where $|\tilde{m}\rangle_{a} \equiv e^{i n_{0}\left(m_{0}-2 m\right) \tau}|m\rangle_{a}$ and $|\tilde{n}\rangle_{f} \equiv$ $e^{i m_{0}\left(n_{0}-2 n\right) \tau}|n\rangle_{f}$ are defined. The orthogonality relations between Schmidt eigenvectors can be satisfied approximately:

$$
\begin{aligned}
& { }_{a}\left\langle u_{k} \mid u_{l}\right\rangle_{a}=\sum_{m=-j}^{j} U_{k}^{*} U_{l} \approx \int d m U_{k}^{*} U_{l}=\delta_{k l} \\
& { }_{f}\left\langle v_{k} \mid v_{l}\right\rangle_{f}=\sum_{n=-\infty}^{\infty} V_{k}^{*} V_{l} \approx \int d n V_{k}^{*} V_{l}=\delta_{k l},
\end{aligned}
$$

provided that $U_{k}$ and $V_{k}$ change smoothly with integer arguments. Therefore Eq. (19) and (20) are limited to modes with low $k$ 's, which is sufficient at short times when the expansion is dominated by low modes.

It is interesting that the widths of the gaussian factor in $U_{k}$ and $V_{k}$ decrease with time, due to the time-dependent scale factor $\xi$. Such a narrowing is not obvious because the left side of Eq. (11) seems to have widths that are constant in time. However, by taking the Fourier transform of the $e^{-x^{2} / \sigma_{A}^{2}-y^{2} / \sigma_{F}^{2}} e^{-i g x y t}$ with respect to $x$, one can see that the transformed function is 'squeezed' in a variable consisting a linear combination of $\tilde{x}$ and $y$, where $\tilde{x}$ is the Fourier variable of $x$. The same feature can also been seen if one takes the Fourier transform with respect to $y$. As $x(y)$ corresponds to atom (photon) number, its Fourier variable may be interpreted as a phase degree of freedom conjugate to the number variable. The narrowing found here therefore reflect a time-dependent number-phase correlation developed between two subsystems [13.

The entanglement entropy can now be calculated in closed form:

$$
\mathcal{S}(t) \approx-\left[\frac{\mu^{2}}{1-\mu^{2}} \ln \left(\mu^{2}\right)+\ln \left(1-\mu^{2}\right)\right]
$$

with the time-dependence of $\mu$ given by Eq. (14). The effective Schmidt number has a simpler expression:

$$
\mathcal{K}(t) \approx \sqrt{1+\left(\sigma_{A} \sigma_{F} \tau\right)^{2}}
$$

which is an increasing function of the dimensionless time $\tau$.

Eq. (21) and (22) describe how the degree of entanglement increases with time. In Fig. 2, we illustrate the time dependence of the entanglement entropy as given by Eq. (21) and compare it with the exact results obtained from numerical Schmidt decomposition. We found a very good agreement in the time interval from zero up to a certain break time $\tau_{B} \approx 1 / \sigma_{\max }$, where $\sigma_{\max }=\max \left\{\sigma_{A}, \sigma_{F}\right\}$. The existence of a break time is understood from the fact that $U_{k}\left(V_{k}\right)$ changes more rapidly with $m(n)$ as time 


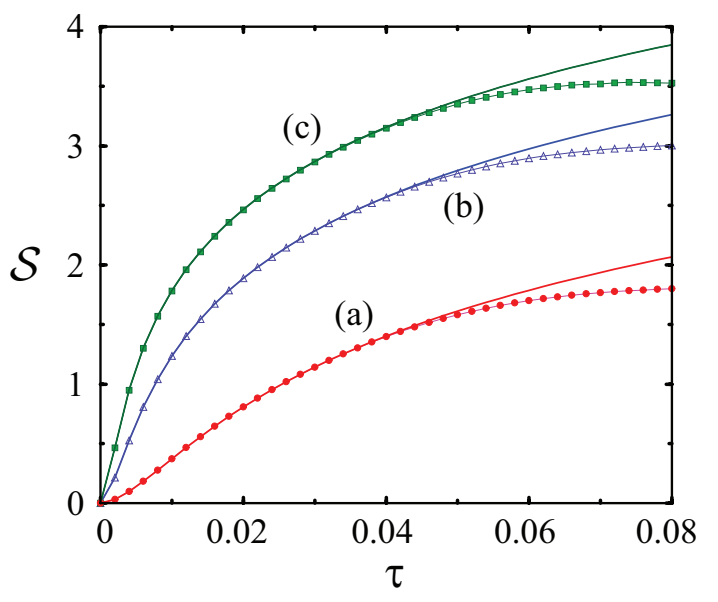

FIG. 2: (Color online) An illustration of the time-dependence of entanglement entropy. The solid lines show the results from Eq. (21), and the lines with data points are obtained by exact numerical Schmidt decomposition. The initial atomic state is an atomic spin coherent state with $N_{A}=2 \sigma_{A}^{2}$ atoms, and the fields are in coherent states. The following parameters are used: (a) $\sigma_{F}=24, \sigma_{A}=3, n_{0}=0$ and $m_{0}=0$. (b) $\sigma_{F}=24, \sigma_{A}=10, n_{0}=12$ and $m_{0}=2$. (c) $\sigma_{F}=24, \sigma_{A}=18$, $n_{0}=0$ and $m_{0}=0$. All the curves are with the same value of $\max \left\{\sigma_{A}, \sigma_{F}\right\}=24$ so that break time $\tau_{B} \approx 0.04$ is the same.

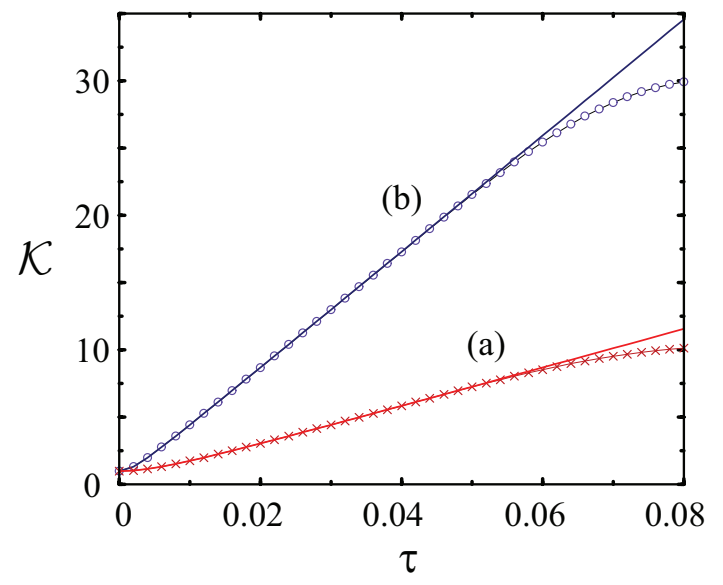

FIG. 3: (Color online) An illustration of time-dependence of effective Schmidt number. The solid lines show the results from Eq. (22), and the line with data points are obtained by exact numerical Schmidt decomposition. The parameters are: (a) $\sigma_{F}=24, \sigma_{A}=6, n_{0}=0$ and $m_{0}=0$. (b) $\sigma_{F}=24$, $\sigma_{A}=18, n_{0}=0$ and $m_{0}=0$.

increases, and hence our continuous variable approximation becomes invalid at long times. Indeed, at the break time $\tau=\tau_{B}$, the width of the gaussian factor $U_{k}$ or $V_{k}$ is of the order 1 , which is minimum separation between integers.

In Fig. 3, we illustrate the time-dependence of the effective Schmidt number $\mathcal{K}(t)$ as given by Eq. (22). We see that $\mathcal{K}(t)$ grows almost linearly with time with the rate $\sigma_{A} \sigma_{F}$. The results also shows a good agreement with exact numerical calculation before the break time. We point out that at $\tau=\tau_{B}, \mathcal{K}$ reaches $\sqrt{1+\sigma_{\text {min }}^{2}}$ ( where $\left.\sigma_{\min }=\min \left\{\sigma_{A}, \sigma_{F}\right\}\right)$, which is independent of the peak positions $n_{0}$ and $m_{0}$.

Having discussed the entanglement in an ideal cavity, let us turn to systems with a leaky cavity. By injecting photons into the cavity, the question is how the output photons are entangled with atoms. According to the Hamiltonian (2), we notice that the interaction effectively shift the resonant frequencies of the cavity. For a given atom number difference $m$, which corresponds to the eigenvalue of $J_{z}$, the frequency shift is $g\left(N_{A} / 2 \pm m\right)$ for the \pm polarizations. We can generalize the inputoutput relations for field operators 14 to account for the shifts, which reads,

$$
a_{ \pm, \text {out }}(\omega ; m)=e^{i 2 \theta_{ \pm}(\omega ; m)} a_{ \pm, \text {in }}(\omega ; m)
$$

where

$$
e^{i 2 \theta_{ \pm}(\omega ; m)}=\frac{\kappa_{c}+i \delta \mp i g m}{\kappa_{c}-i \delta \pm i g m} .
$$

Here we have defined $\delta=\omega-\omega_{c}-g N_{A} / 2$, with $\omega_{c}$ being the resonant frequency of the cavity, and the cavity field decay rate $\kappa_{c}$. Note that the detuning $\delta$ has included the term $g N_{A} / 2$.

In the bad cavity limit $\kappa_{c} \gg g m$, we find that $\theta_{ \pm}(\omega ; m)$ takes a simple form:

$$
\theta_{ \pm}(\omega ; m) \approx \theta_{0}(\omega) \mp \frac{g m}{\kappa_{c}} .
$$

provided that $\kappa_{c}^{2} \gg \delta^{2}$. Here $\theta_{0}(\omega)=\tan ^{-1}\left(\delta / \kappa_{c}\right)$ is a phase angle independent of $m$, and the second term $g m / \kappa_{c}$ is independent of $\omega$. Eq. (25) indicates that if the input field frequency $\omega$ is near $\omega_{c}+g N_{A} / 2$ compared with the cavity width, then the output field operator would pick up the phase angle $\mp g m / \kappa_{c}$, in addition to $\theta_{0}(\omega)$. Such a result can be translated into Schrodinger picture. Suppose the input field is described by a single mode in the Fock state $\left|N_{+}, N_{-}\right\rangle_{\text {in }}$ and the atomic states is the state $|m\rangle_{a}$, then we have the transformation for the input-output state vectors [15]:

$$
\left|N_{+}, N_{-}\right\rangle_{\text {in }}|m\rangle_{a} \rightarrow e^{i 2 \theta_{0}(\omega) s-i 2 g m n / \kappa_{c}}\left|N_{+}, N_{-}\right\rangle_{\text {out }}|m\rangle_{a}
$$

where $n=N_{+}-N_{-}$and $s=N_{+}+N_{-}$. Apart from an unimportant factor $e^{i 2 \theta_{0}(\omega) s}$ that is not responsible for quantum entanglement, the correlated phase factor $e^{-i 2 g m n / \kappa_{c}}$ is precisely of the same form as Eq. (6) derived in an ideal cavity. Therefore our Schmidt analysis in the ideal cavity can be employed by taking the interaction time $t=2 / \kappa_{c}$, which is the finite life time of cavity fields. In particular, if $\tau_{B} \geq 2 / \kappa_{c}$, we have

$$
\mathcal{K} \approx \sqrt{1+\left(2 \sigma_{A} \sigma_{F} g / \kappa_{c}\right)^{2}}
$$

after all the input photons are fully converted into the output mode. 
It is interesting to employ Eq. (27) to estimate the entanglement generated in free space, i.e., without the cavity. This may be done by replacing $g$ with the corresponding value in free space $g_{f}$, and $2 / \kappa_{c}$ by the (single pass) interaction time $t_{f}$. Apparently, $g>g_{f}$ due to the cavity enhancement would increase the entanglement for a fixed interaction time. A crude analysis by Kuzmich et al. in Ref. 1] suggested that $g_{f}$ is inversely proportional to the volume defined by the spatial extend of the atoms in free space. As $g$ is inversely proportional to the cavity mode volume, $g / g_{f}$ could be approximated by the volume ratio. However, a rigorous analysis in free space is difficult because of the involvement of multi-mode dynamics in general [1].

To conclude, we perform the Schmidt analysis of the photon-atom entanglement generated by Faraday rotation in a cavity. We present an approximate analytic formula of Schmidt decomposition, which reveals the Schmidt mode structures and the time-dependence of entanglement. In particular we show that the initial fluctuations $\sigma_{F}$ and $\sigma_{A}$ are key parameters to control the rate of change of entanglement. This work is restricted to the class of gaussian amplitudes defined by Eq. (9) and Eq. (10), which covers a range of quantum states of photons and atoms that have a well defined peak and width. In this paper we also discuss the entanglement in leaky systems when cavity decay rate is sufficiently large. The quantum dynamics beyond the bad cavity limit is out of the scope of this paper. This problem is difficult because the phase shift of output photons becomes a nonlinear function of $m$ when $\kappa_{c}$ is comparable with $g$. The nonlinearity, which comes from the competition between natural cavity response function and atom-field interaction, cannot be treated by the approximation method described in this paper. This is an open topic for future investigations.

This work is supported in part by the Research Grants Council of the Hong Kong Special Administrative Region, China (Project No. 401305).

[1] A. Kuzmich, N. P. Bigelow, and L. Mandel, Europhys. Lett. 42, 481 (1998); A. Kuzmich et al., Phys. Rev. A 60, 2346 (1999); A. Kuzmich, L. Mandel, and N. P. Bigelow, Phys. Rev. Lett. 85, 1594 (2000).

[2] Y. Takahashi et al., Phys. Rev. A 60, 4974 (1999); Lars
Bojer Madsen and K. Molmer, Phys. Rev. A 70, 052324 (2004); M. Takeuchi et al., Phys. Rev. Lett. 94, 023003 (2005); C. Genes and P. R. Berman, Phys. Rev. A 73, 013801 (2006).

[3] Daniel Oblak et al., Phys. Rev. A 71, 043807 (2005).

[4] A. Kuzmich and E. S. Polzik, Phys. Rev. Lett. 85, 5639 (2000); K. Hammerer, E. S. Polzik, and J. I. Cirac, Phys. Rev. A 72, 052313 (2005).

[5] C. Schori, B. Julsgaard, J. L. Sorensen, and E. S. Polzik, Phys. Rev. Lett. 89, 057903 (2002); T. Opatrny and J. Fiurasek, Phys. Rev. Lett. 95, 053602 (2005).

[6] S. Massar and E. S. Polzik, Phys. Rev. Lett. 91, 060401 (2003).

[7] S. Dasgupta, A. Biswas, and G. S. Agarwal, Phys. Rev. A 71, 012333 (2005).

[8] For an overview of Schmidt decomposition, see, A. Ekert and P. L. Knight, Am. J. Phys. 63, 415 (1995) and references therein.

[9] K. Hammerer et al., Phys. Rev. A 70, 044304 (2004).

[10] R. Grobe, K. Rzążewski and J. H. Eberly, J. Phys. B 27, L503 (1994); W.-C. Liu, J. H. Eberly, S. L. Haan and R. Grobe, Phys. Rev. Lett. 83, 520 (1999); R. E. Wagner, P. J. Peverly, Q. Su, and R. Grobe, Laser Phys. 11, 221 (2001).

[11] Spin coherent states are defined by $|\xi\rangle=(1+$ $\left.|\xi|^{2}\right)^{-j} e^{\xi J_{+}}|j,-j\rangle$, see F. T. Arecchi, E. Courtens, R. Gilmore, and H. Thomas, Phys. Rev. A 6, 2211 (1972). The amplitudes of states can be well approximated by a Guassian with $\sigma_{A}=\sqrt{N_{A} / 2}$ and $m_{0}=$ $N_{A}\left(|\xi|^{2}-1\right) / 2\left(|\xi|^{2}+1\right)$ when $N_{A} \gg 1$.

[12] For a two-mode coherent field state $\left|\alpha_{+}\right\rangle\left|\alpha_{-}\right\rangle$,

$$
P_{s, n}=e^{-\langle s / 2\rangle} \frac{\left(\alpha_{+}\right)^{s / 2+n / 2}\left(\alpha_{-}\right)^{s / 2-n / 2}}{\sqrt{(s / 2+n / 2) !(s / 2-n / 2) !}}
$$

where $\langle s\rangle=\left|\alpha_{+}\right|^{2}+\left|\alpha_{-}\right|^{2}$ is the average total photon number. When $\langle s\rangle \gg 1, F_{n}$ defined after Eq. (5) can be well approximated by a gaussian with $\sigma_{F}=2 \sqrt{\langle s\rangle}$ and $n_{0}=\left|\alpha_{+}\right|^{2}-\left|\alpha_{-}\right|^{2}$.

[13] K. W. Chan and J. H. Eberly (unpublished).

[14] See for example, D. F. Walls and G. J. Milburn, Quantum Optics, Springer, Berlin (1994).

[15] A single mode field considered here does not necessarily mean that it is purely monochromatic. In general a (wave packet) mode corresponds to a general superposition of frequency modes, so that the input Fock state $\left|N_{ \pm}\right\rangle_{\text {in }}=b_{ \pm, \text {in }}^{\dagger N}|0\rangle / \sqrt{N_{ \pm}}$! is defined by the operator: $b_{ \pm, \text {in }}=\int f(\omega) a_{ \pm, \text {in }}(\omega) d \omega$. A similar definition can be written for the output mode. For the state transformation (26) to be valid, $f(\omega)$ has to concentrated at $\omega_{c}+g N_{A} / 2$ and its width is small compared with $\kappa_{c}$. 